Driesenaar, J.A., Dulmen, S. van, Weert, J.C.M. van, Noordman, J. Patients' evaluation of a preparatory online communication tool for older patients with cancer preceding chemotherapy. Cancer Nursing: 2020, 43(2), p. E71-E78

Postprint version

Journal website

: https://insights.ovid.com/crossref?an=00002820-900000000-99089

Pubmed link

DOI

: https://www.ncbi.nlm.nih.gov/pubmed/30433895

: $\quad 10.1097 /$ NCC. 0000000000000660

This is a Nivel certified Post Print, more info at nivel.nl

\title{
Patients' Evaluation of a Preparatory Online Communication Tool for Older Patients With Cancer Preceding Chemotherapy
}

\author{
Jeanine A. Driesenaar, MSc, Sandra van Dulmen, PhD, Julia C. M. van \\ Weert, PhD, Janneke Noordman, PhD
}

Author Affiliations: Netherlands Institute for Health Services Research, Utrecht (Ms Driesenaar and Prof van Dulmen and DrNoordman); and Department of Primary and Community Care, Radboud University Medical Center,Nijmegen (Dr van Dulmen), the Netherlands; Faculty of Health Sciences, University College of Southeast Norway, Drammen (Prof van Dulmen); and Amsterdam School of Communication Research, Department of Communication Science, University of Amsterdam, the Netherlands (Prof van Weert).

Background: The online tool PatientVOICE has been developed to enhance older patients' participation during educational nursing encounters preceding chemotherapy and to increase their information recall. Objective: The aim of this study was to evaluate perceived usefulness, usability, satisfaction with emotional support, language use, attractiveness, and visit intention of PatientVOICE by older (ex-)patients with cancer. Methods: Older (ex-)patients with cancer were invited to evaluate the website via an online questionnaire. Perceived usefulness, usability, and satisfaction with emotional support were measured using evaluation statements, the System Usability Scale, and an adapted subscale of the Website Satisfaction Scale, respectively. Questions were also included about language use and attractiveness of the website and patients' intention to visit the website. Results: A total of 44 questionnaires were analyzed. Many patients evaluated the provided information and other integrated techniques (such as the question prompt sheet, video fragments, and the audio facility) positively on aspects as usefulness and helpfulness. The usability was considered good (mean scale score, 74.3). Most patients (84.9\%) considered the language use on the website clear, and $63.6 \%$ of the patients found the website attractive. Many patients $(71.9 \%)$ would visit the website if they would like to gather information on the encounter preceding chemotherapy, and $62.5 \%$ of the patients would do this for information about chemotherapy. Conclusions: PatientVOICE is evaluated as a useful and user-friendly tool, enabling patients to prepare themselves for the nursing encounter preceding chemotherapy and to gather information about chemotherapy. Implications for Practice: Preparatory online 
Driesenaar, J.A., Dulmen, S. van, Weert, J.C.M. van, Noordman, J. Patients' evaluation of a preparatory online communication tool for older patients with cancer preceding chemotherapy. Cancer Nursing: 2020, 43(2), p. E71-E78

tools, such as PatientVOICE, can be implemented in hospitals to offer patients extra support.

Prior to starting chemotherapy, patients with cancer have an encounter with their oncology nurse (or oncologists) in which complex and elaborate information about the treatment and its possible adverse effects is provided. These encounters pose many challenges, especially for older patients (aged $\geq 65$ years). In general, older patients' participation during interactions with healthcare providers is rather low1-3; many patients experience barriers to participate, lack sufficient skills to retrieve relevant information, 4 and are reserved in expressing their needs and preferences.2,5 In addition, older patients' information recall is often low. Jansen et al6 showed that of the information provided during educational nursing encounters less than a quarter of the advices on how to handle adverse effects are recalled.

Besides, because of aging-related deficiencies (eg, memory loss, hearing and vision problems, comorbidity, a smaller social network), older patients are more vulnerable in their interaction with healthcare providers.7,8 This requires sensitive and effective communication of healthcare providers, whereby it is essential to take the patients' needs into account. Such a patient-centered, tailored approach helps patients to cope better with having cancer, its treatment, and adverse effects.2,9-11 With the purpose to enhance older patients' participation during educational nursing encounters and increase their information recall, van Weert et al12 developed in the VOICE study a preparatory paper brochure for older patients and changed the structure of the encounter in the Netherlands. This brochure "Talking About Chemotherapy" describes the aims and topics of the nursing encounter and contains a question prompt sheet (QPS) to prepare questions for the oncology nurse. A thorough evaluation showed that, in the prepared encounters, nurses discussed realistic expectations more frequently, and patients askedmore questions.12 Only minor improvements in patients' information recall were found, which might be due to insufficient tailoring of the preparatory information or communication to the patients' needs.

In a follow-up study, we developed a more elaborate, online version of the preparatory paper brochure called "PatientVOICE" using the intervention mapping framework.13 We opted for a webbased intervention, as the Internet serves as an important source for information and support for patients with cancer. A recent study showed in a small sample that older cancer patients consider online health information very useful,14 and it has been found that online health information is promising in facilitating immediate, intermediate, and long-term health outcomes in older patients.15 Furthermore, it is important to pay (more) attention in literature to the development, evaluation, and existence of this relatively new type of interventions and to share this knowledge with patients, families, and healthcare professionals, as this is useful for the development and the implementation of this kind of tools.

PatientVOICE (www.chemowijzer.nl) consists of 3 sections (Table 1): (1) a section with information on the nursing encounter preceding chemotherapy, (2) a section with information about chemotherapy, and (3) a secured personal page for patients.

Several "techniques," which are based on scientific literature, were integrated into the sections of PatientVOICE. First, Patient- VOICE provides precounseling information to prepare patients sufficiently on the nursing encounter and on chemotherapy.

Second, a QPS has been integrated, a list of 15 designed questions on which patients can tick which topics they would like to discuss during the encounter. Question prompt sheets are found to improve patient question asking and participation.16 Third, PatientVOICE makes use of video-modeling. Three short video fragments are available to show patients "best practices" about communication with their healthcare provider. One video is about preparing the encounter, the second is about expressing needs and concerns, and the third shows tips about audio recording the encounter. Last, an audio 
Driesenaar, J.A., Dulmen, S. van, Weert, J.C.M. van, Noordman, J. Patients' evaluation of a preparatory online communication tool for older patients with cancer preceding chemotherapy. Cancer Nursing: 2020, 43(2), p. E71-E78

facility is integrated into the secured personal page, enabling patients to listen back to their consultation, which aims to improve their information recall. See the Figure for a screenshot of the homepage of PatientVOICE.

Optimal perceived usefulness and usability of a website are prerequisites for people to make use of a website. Perceived usefulness refers to "the degree to which a person believes that using a particular system would enhance his or her job performance,"17 and usability addresses "the extent to which a product can be used by specified users to achieve specified goals with effectiveness, efficiency, and satisfaction."18 This study aimed to get insight into the evaluation of the perceived usefulness and usability of PatientVOICE among older (ex-)patients with cancer. Also, satisfaction with emotional support, language use, attractiveness, intention to visit PatientVOICE, and suggestions for improvement of the website have been assessed.

\section{Methods}

We used a cross-sectional design to explore the perceived usefulness and usability of the online preparatory tool PatientVOICE among older (ex-)patients with cancer using questionnaires. This study is part of a larger research and development project PatientVOICE. The Committee on Research Involving Human Subjects Arnhem-Nijmegen decided that the study protocol did not fall within the remit of the Law on Medical Scientific Research Involving Human Beings (2014-1431).

\section{[table 1]}

\section{Procedure}

Patient recruitment for this study took place via 3 different ways between February 2016 and June 2016. First, 12 hospitals that had been involved in the previous VOICE12 and PatientVOICE13 studies were approached for participation in this study, of which 6 agreed. Patients were recruited through these 6 participating hospitals by leaflets, which were put in the waiting rooms of the hospitals or were handed out to eligible patients by oncology nurses and medical assistants. Brief information was stated on the leaflet about the website, the purpose of the study, inclusion criteria, and the link to an online evaluation questionnaire.

Because of a low participation rate of patients in the 6 hospitals, we decided to recruit patients via another way as well. Criteria for participation were adapted (see Sample and Inclusion Criteria). Eligible patients were invited for participation via the patient panel PanelCom (www.panelcom.nl). PanelCom is a panel hosted by the University of Amsterdam (collaboration between the Departments of Communication Science and Medical Psychology in the Academic Medical Center) and consists of a group of approximately 700 participants, mainly (ex-)cancer patients, who participated in previous University of Amsterdam studies and consented to be contacted again to participate in future studies. The coordinator of PanelCom sent an e-mail with information about the study and the link to the online questionnaire to eligible patients. In addition, older (ex-)patients, who had been involved in a previous study of our research institute NIVEL (Netherlands Institute for Health Services Research) and had registered for participation in future projects at our institute were sent an invitational e-mail. Before study participation, all patients have been informed about the study background and procedure. Patients consented with study participation by filling in the online questionnaire (or did not consent by not filling in the questionnaire). The data were anonymously collected and analyzed according to the Dutch privacy regulations.

\section{Sample and Inclusion}

Criteria Inclusion criteria differed per procedure. Regarding the patient recruitment through hospitals, patients were eligible if they were 65years or older and were being treated with chemotherapy or had been treated in the preceding 5 years with chemotherapy. 
Driesenaar, J.A., Dulmen, S. van, Weert, J.C.M. van, Noordman, J. Patients' evaluation of a preparatory online communication tool for older patients with cancer preceding chemotherapy. Cancer Nursing: 2020, 43(2), p. E71-E78

Other criteria were applied during the recruitment via PanelCom; patients were eligible if they were aged between 65 and 69years and if they had cancer or were cured of cancer.

To include as many patients as possible, not only patients who were treated with chemotherapy were approached, but also those who had cancer or were cured of cancer and had never been treated with chemotherapy. Patients in the panel of 70years or older had already been invited for another study and were therefore excluded from participation in this study to avoid overload; 169 patients were approached.

In addition, (former) patients with cancer who were involved in a previous NIVEL study and had agreed to be contacted to participate in future studies were eligible if they were 65years or older and if they had cancer or were cured of cancer. Six (former) patients were invited. As an incentive for participation, 2 gift vouchers (to the value of $€ 20$ ) were raffled among the participants.

\section{[figure 1]}

\section{Instrument: Online Questionnaire}

An online questionnaire was used to evaluate PatientVOICE.

PatientVOICE is primarily developed for patients scheduled for chemotherapy, and not especially for patients who already are treated, or have been treated, with chemotherapy. Therefore, participating patients were instructed to look at the website as if they would receive chemotherapy for the first time.

Before completing the questions, patients were asked to visit the website and look at the different sections on the website to get a general impression of PatientVOICE. Then, each section (and some subsections) was evaluated separately. Patients received the instruction to go to a specific page and, depending on the type of (sub)section, were asked, for instance, to read the information, watch video fragments, or fill in the QPS. Subsequently, patients answered questions about that particular section. After completing these questions, patients were instructed to go to thenext sectionon PatientVOICE. Completion of the questionnaire, including viewing the website, took approximately 30 to 40 minutes.

\section{Variables}

In the following paragraphs, we describe the included variables in the questionnaire in more detail: patients' characteristics, perceived usefulness of the website, usability of the website, satisfaction with emotional support, language use and attractiveness, intention to visit the website, and completeness of the information.

\section{Patients' Characteristics}

The questionnaire assessed patients' sociodemographic characteristics (ie, sex, age, educational level, ethnic background), type of cancer, chemotherapy, and their skills and experience with Internet and computer.

\section{Perceived Usefulness of the Website}

To assess the perceived usefulness of the website, the different sections of the website have been evaluated separately. With respect to the sections "encounter preceding chemotherapy" and "chemotherapy," statements were formulated to measure the perceived usefulness of these sections. The statements were about whether patients found the sections useful, easy to understand, helpful, reliable, reassuring, upsetting, confusing, timely, helpful, too elaborate, and complete. These statements are based on a study by Butow et al.19,20 For the evaluation of other sections, we supplemented statements to measure other aspects of the usefulness relevant to that section. Regarding the QPS, patients had to indicatewhether they considered the QPS useful, redundant, and easy to complete and whether it Statements were also formulated about whether the video fragments 
Driesenaar, J.A., Dulmen, S. van, Weert, J.C.M. van, Noordman, J. Patients' evaluation of a preparatory online communication tool for older patients with cancer preceding chemotherapy. Cancer Nursing: 2020, 43(2), p. E71-E78

are useful, redundant, and realistic; or give a good example of communicating with a nurse; or trigger emotional responses; and whether or not patients recognize themselves in the patient in the video fragments.

Regarding the audio facility, patients were asked about the usefulness, helpfulness, and value of an audio recording and whether it would help them remember what awaits them. Patients answered on a 4-point Likert scale whether they totally disagree, disagree, agree, or totally agree with a statement.

\section{Usability of the Website}

The usability of the website was measured using the System Usability Scale (SUS). The SUS is a reliable scale for measuring usability, including 10 items about several facets of usability, such as the complexity of the website and the ease of using it.21 Items were scored on a 5-point Likert scale, ranging from 0 (strongly disagree) to 4 (strongly agree). System Usability Scale scores were calculated using the scoring instructions.21 System Usability Scale scores range between 0 and 100 . Higher scores indicate enhanced usability.

\section{Satisfaction With Emotional Support}

Satisfaction with emotional support was assessed using a subscale of the Website Satisfaction Scale (WSS).22 This scale consists of 4 items (eg, "the website gives me self-confidence"), which were scored on a 5-point Likert scale ranging from 1 (strongly disagree) to 5 (strongly agree). The original scale has a 7-point Likert scale, which we changed into a 5-point Likert scale to make it more consistent with the other 5-point scales we used in the questionnaire. A mean score of the 4 items was calculated, ranging between 1 and 5, with a scale midpoint of 3 .

Higher scores refer to greater satisfaction. The reliability of the scale was high in our sample of patients (Cronbach's !=.93).

\section{Language Use and Attractivenesss}

The clearness of the language use and the attractiveness of the website were assessed by 2 single statements and were scored on a 5-point Likert scale ranging from 1 (strongly disagree) to 5 (strongly agree).

\section{Intention to Visit the Website and Suggestions for Improvement of the Website}

Open-ended questions were used to assess whether patients would visit the website if they wanted information about the nursing encounter and chemotherapy and whether they missed information in general and on practical advices about the daily life during chemotherapy. Additional remarks or suggestions for improvement were assessed by open-ended questions.

Data Analysis Data analysis was conducted using Stata 14.0 (StataCorp LP, College Station, Texas). Descriptive statistics were used to describe the characteristics of the participants (means, SDs, and percentages) and to analyze the results (means, SDs, and percentages). Open coding was used to analyze the comments regarding open-ended questions.

n Results Study Sample The online questionnaire was opened 85 times from 66 unique IP addresses. A total of 44 questionnaires were completed sufficiently to report results. Of these, 31 questionnaires were completed totally, and 13 were completed partially. Table 2 shows the participants' characteristics.

Perceived Usefulness of the Website Below, the main results on the perceived usefulness of the website have been described.

Almost all patients $(97.7 \%)$ considered the information on the section "encounter preceding chemotherapy" useful. An overwhelming majority of the patients (range, 79.6\%Y95.5\%) (totally) agreed with the statements that the information is easy to understand, helpful to themselves, reliable, reassuring, timely, and helpful to family and friends (Table 3 ). 
Driesenaar, J.A., Dulmen, S. van, Weert, J.C.M. van, Noordman, J. Patients' evaluation of a preparatory online communication tool for older patients with cancer preceding chemotherapy. Cancer Nursing: 2020, 43(2), p. E71-E78

With respect to the section "chemotherapy," all patients (100\%) indicated that the information was easy to understand, and $97.1 \%$ of the patients found it useful. A large majority (range, $73.5 \%$ Y94.1\%) considered the information helpful, reliable, reassuring, helpful to family and friends, and complete (Table 3).

Regarding the usefulness of the audio-recording facility, nearly three-quarters $(73.5 \%)$ of the patients (totally) agreed with the statement that an audio recording would be valuable and that it would be helpful to family and friends. Approximately two-thirds (64.7\%) answered that an audio recording would be helpful to themselves and that it would help them remember what awaits them (Table 3). The QPS was considered by a vast majority (range, 95.2\% 97.6\%) as useful and easy to fill out and that completing the QPS would help them to ask their questions in the nursing encounter.

Few patients (7.1\%) considered the QPS redundant (Table 4).

With respect to the evaluation of the video fragments on the website, of the 44 patients who filled in the questionnaire, 34 patients (77.3\%) watched 1 or more video fragments. Most patients (range, $79.4 \%$ Y $85.3 \%$ ) thought the video fragments are useful and realistic and that they give a good example of how to communicate with their nurse (Table 4).

Usability of the Website, Emotional Support, Language Use, and Attractiveness Patients had a mean SUS score of 74.3 (SD, 13.8; range, 42.5Y97.5; $n=33$ ), which indicates good usability.23 Regarding satisfaction with emotional support, the mean WSS score was 2.8 (SD, 0.92; range, 1Y4.5; $n=32$ ), indicating that patients were not satisfied with the emotional support. Most patients (84.9\%) considered the language use on the website clear, and $63.6 \%$ of the patients found the website attractive.

\section{[table 2]}

Intention to Visit the Website A majority of the patients (71.9\%) would visit the website if they would need to prepare themselves for an encounter preceding chemotherapy. If patients would look for information about chemotherapy, $62.5 \%$ of the patients would visit the website. Patients who "not" or "maybe" would visit the website were asked for their reasons using open-ended questions. They provided the following reasons: receiving enough information from the hospital or Internet (in general), preferring (first) to receive information about chemotherapy from the healthcare provider, not being in the mood to look on Internet, having no need for support, looking for information on other websites themselves, it depends on the situation (whether you have questions or already have received information or the type of guidance), and it depends on whether healthcare providers inform you about the website.

Improvements of the Website More than three-quarters of the patients (77.4\%) indicated that the website provides enough practical advices about the daily life during chemotherapy. Some patients noted that it is impossible to provide exhaustive information, because every patient is different. Patients who answered that the website did not provide enough practical advices would like more extensive information about the provided practical advices (eg, physical activity, adverse effects, and taking care of yourself). One patient mentioned that it is not possible for online tools (in general) to support patients regarding acceptation and coping with emotions, which are also important aspects in daily life.

In addition, some patients (38.7\%) missed "other information" on the website. Patients mentioned to prefer (more) information about toilet use, cleaning, (a clearer explanation of) disposing contaminated garbage, how to tell it to one's employer, and precautions at work. Some patients missed depth and details, and 1 patient preferred that patients' perceptions could be more taken into account.

\section{[table 3]}

Discussion In a previous study, we have developed an elaborated and online version of the brochure "Talking About Chemotherapy."13 We have extended the brochure with information based on the 
Driesenaar, J.A., Dulmen, S. van, Weert, J.C.M. van, Noordman, J. Patients' evaluation of a preparatory online communication tool for older patients with cancer preceding chemotherapy. Cancer Nursing: 2020, 43(2), p. E71-E78

results of a needs assessment in this previous study, in order to correspond it with patients' needs. In comparison to the brochure, PatientVOICE provides more information and supportive aids than the brochure. It provides information on chemotherapy and more preparatory information about the encounter preceding chemotherapy, supports patients with video fragments, and offers an audio facility.

\section{[table 4]}

Our results indicate that older patients value this extended information and facilities on PatientVOICE, as they evaluated PatientVOICE as a useful and user-friendly tool. Most patients considered the provided information about the encounter preceding chemotherapy and chemotherapy as useful, easy to understand, helpful, reliable, reassuring, timely, helpful, and complete. Other integrated techniques (ie, the QPS, video fragments, and the audio facility) were also evaluated positively on several aspects such as usefulness and helpfulness. The usability of PatientVOICE, assessed with the SUS, was considered good. In addition, it indicates that we developedVby using the intervention mapping frameworkVa tool that is tailored to patients' needs. Our findings are in line with other online interventions that have integrated similar techniques and were evaluated positively or effectively (see, for example, Albada et al20 and van Bruinessen et al24).

Although patients were positive about PatientVOICE, results showed that they were not clearly satisfied or dissatisfied with its emotional support. Satisfaction with emotional support is defined as the extent to which patients feel that the tool helps with coping with emotions and stress. The mean WSS score (2.8) was around the scale midpoint of 3, indicating that patients do not experience much emotional support and that PatientVOICE does not fulfill patients' affective needs (ie, regarding coping with emotions, stress, and confidence) sufficiently.

A possible explanation is that PatientVOICE concentrates particularly on providing information, which is aimed at fulfilling the patients' informational needs and which was also the main purpose of the tool. Therefore, it is still very important that healthcare providers are responsive to the patients' need for emotional support. However, Brandes et al25 found that an interplay between "instrumental concerns" and "emotions" exists.

"Instrumental concerns" are related to information provision, such as information overload, receiving insufficient of incorrect information, and difficulties with searching, finding, and judging of information, whereas emotions refer to feelings such as fear and frustration. As an interplay between them exists, it might be possible that affective needs might be fulfilled by fulfilling informational needs. Future research might take a closer look at this.

Furthermore, the results indicate that PatientVOICE might be more suited for patients who want to prepare themselves for the encounter preceding chemotherapy than for patients who are seeking only information about chemotherapy $(71.9 \%$ of the patients would visit the website for information on the encounter, whereas $62.5 \%$ would do this for information on chemotherapy).

A possible explanation for this finding is that there is already ample reliable information available about chemotherapy on the Internet (eg, provided by the patients' hospitals and the Dutch Cancer Society). However, there is less online information supporting patients to prepare themselves for the encounter. Patient- VOICE distinguishes itself from most other online tools regarding this aspect.

Although the aim of the larger study was to develop a tool to support especially older cancer patients, the tool might be very useful for younger patients who are going to receive chemotherapy as well. As this study performed an evaluation only of the perceived usefulness and usability, future research should focus on the effectiveness of PatientVOICE on patient participation during interactions with oncology nurses, information recall, and other outcomes (eg, self-efficacy). Examining which specific elements and techniques of the website are effective or less effective (eg, by analyzing user-statistics in combination with outcomes) might provide important insights for improvement of the tool and for developing other online tools for patients with cancer as well. 
Driesenaar, J.A., Dulmen, S. van, Weert, J.C.M. van, Noordman, J. Patients' evaluation of a preparatory online communication tool for older patients with cancer preceding chemotherapy. Cancer Nursing: 2020, 43(2), p. E71-E78

With respect to the implementation of the tool, it is important that healthcare providers inform patients about the existence of the website (during a consultation and/or through folders), preferably some time before the nurse encounter preceding chemotherapy to ensure that patients have some time to visit the website. Future research can also pay attention to successful implementation of the tool on a large scale, to make it accessible formany (hospital) patients.

Furthermore, the patients' comments about improvements of the website have been taken into account, and some changes to the website have been made (eg, more information about cleaning). In this study, we have evaluated a Dutch online tool.

However, the results might be useful and relevant at international level as well. As our results indicate, a tool such as Patient- VOICE can be very valuable to offer to patients by hospitals and healthcare providers in preparation to chemotherapy. Patient- VOICE consists of multiple useful elements and techniques for a preparatory online communication tool for (older) patients with cancer preceding chemotherapy (Table 1). These techniques can be used in online tools in other countries as well and can be adapted to the cancer care of a specific country. Last, our findings provide insights that can be used for future (international) research aimed at supporting older patients with cancer using online interventions.

\section{Limitations}

The sample of patients consisted for the greatest part of patients who already have been treated with chemotherapy and not of patients who were going to start. This might limit the generalizability of the outcomes.We instructed the patients to look at the website as if they would receive chemotherapy for the first time. Despite this instruction, patients who already have been treated with chemotherapy might have evaluated the website differently than patients who are going to receive chemotherapy in the (near) future, as both groups might have different (emotional and/or informational) needs and preferences. However, we expect that the patients who are going to receive chemotherapy for the first time have a higher need for information than patients who already have been treated; therefore, the website will probably be more valuable for them and might be evaluated more positively than in this study.

Another limitation is the bias of participating patients who are more engaged in research. Most of the patients were recruited through PanelCom. This panel consists of patients who were previously involved in research and consented to be approached for the participation in other studies. These patients could have other needs regarding information on chemotherapy or the nursing encounter than patients who are less engaged in research. This could also limit generalizability of our results, although these patients might have a more critical view, resulting in a thorough evaluation of PatientVOICE.

\section{Conclusions}

Older (former) patients with cancer evaluated the online tool PatientVOICE as a useful and userfriendly tool, which can be used to prepare themselves for the educational visit preceding chemotherapy and also to find information about chemotherapy.

The information on the website and integrated supportive aids such as the question prompt sheet, video fragments, and the audio facility were considered useful by patients. Regarding implementation of the tool, it is important that healthcare providers pay attention to the tool so that patients are aware of its existence. This positive evaluation indicates that the tool can offer much support to patients and their families.

\section{ACKNOWLEDGMENTS}

The authors thank the patients, oncology nurses, and medical assistants for their participation in this study. 
Driesenaar, J.A., Dulmen, S. van, Weert, J.C.M. van, Noordman, J. Patients' evaluation of a preparatory online communication tool for older patients with cancer preceding chemotherapy. Cancer Nursing: 2020, 43(2), p. E71-E78

\section{References}

1. Jansen J, vanWeert JC, de Groot J, van Dulmen S, Heeren TJ, Bensing JM. Emotional and informational patient cues: the impact of nurses' responses on recall. Patient Educ Couns. 2010;79(2):218-224.

2. Posma ER, van Weert JC, Jansen J, Bensing JM. Older cancer patients' information and support needs surrounding treatment: an evaluation through the eyes of patients, relatives and professionals. BMC Nurs. 2009;8:1.

3. Baile WF, Aaron J. Patient-physician communication in oncology: past, present, and future. Curr Opin Oncol. 2005;17(4):331-335.

4. Rademakers J. Kennissynthese: gezondheidsvaardigheden: niet voor iedereen vanzelfsprekend. [Knowledge Synthesis. Health Skills: Not for Everyone]. Utrecht, the Netherlands: NIVEL; 2014. 5. Puts MT, Papoutsis A, Springall E, Tourangeau AE. A systematic review of unmet needs of newly diagnosed older cancer patients undergoing active cancer treatment. Support Care Cancer. 2012;20(7):1377-1394.

6. Jansen J, vanWeert J, van derMeulenN, van Dulmen S, Heeren T, Bensing J. Recall in older cancer patients: measuring memory for medical information. Gerontologist. 2008;48(2):149Y157. 7. Kessels RP. Patients' memory for medical information. J R Soc Med. 2003; 96(5):219-222. 8. Williams SL, Haskard KB, DiMatteo MR. The therapeutic effects of the physician-older patient relationship: effective communication with vulnerable older patients. Clin Interv Aging.

2007;2(3):453-467.

9. Epstein RM, Street RL. Patient-Centered Communication in Cancer Care: Promoting Healing and Reducing Suffering. Bethesda, MD: National Cancer Institute; 2007.

10. Little P, Everitt H, Williamson I, et al. Preferences of patients for patient centred approach to consultation in primary care: observational study. BMJ. 2001;322(7284):468-472.

11. van Koerten C, Feytens M Jansen J, van Dulmen S van Weert J. Communicatiebehoeften van pati:nten met kanker bij aanvang van een behandeling met chemotherapie: een onderzoek naar de rol van curatief of palliatief behandeldoel, leeftijd en geslacht. [Communication needs of cancer patients starting with chemotherapy: a study into the role of curative or palliative treatment goal, age and gender]. Verpleegkunde. 2011;26(1):4-12.

12. Van Weert JC, Jansen J, Spreeuwenberg PM, van Dulmen S, Bensing JM. Effects of communication skills training and a question prompt sheet to improve communication with older cancer patients: a randomized controlled trial. Crit Rev Oncol Hematol. 2011;80(1):145-159. 13. van Dulmen S, Driesenaar JA, Weert JC, van Osch M, Noordman J. PatientVOICE: development of a preparatory, pre-chemotherapy online communication tool for older patients with cancer. JMIR Res Protoc 2017; 6(5):e85.

14. Bolle S, Romijn G, Smets EM, Loos EF, Kunneman M, van Weert JC. Older cancer patients' user experiences with web-based health information tools: a think-aloud study. J Med Internet Res. 2016;18(7):e208.

15. Bolle S, van Weert JC, Daams JG, Loos EF, de Haes HC, Smets EM. Online health information tool effectiveness for older patients: a systematic review of the literature. J Health Commun.

2015;20(9):1067-1083.

16. Brandes K, Linn AJ, Butow PN, van Weert JC. The characteristics and effectiveness of Question Prompt List interventions in oncology: a systematic review of the literature.

Psychooncology 2015;24(3):245-252.

17. Davis FD. Perceived usefulness, perceived ease of use, and user acceptance of information technology. MIS Quarterly. 1986;13(3):319-340.

18. ISO 9241-11. Ergonomic Requirements for Office Work With Visual Display Terminals

(VDTs)VPart 11: Guidance on Usability International Organization for Standardization. 1998. 
Driesenaar, J.A., Dulmen, S. van, Weert, J.C.M. van, Noordman, J. Patients' evaluation of a preparatory online communication tool for older patients with cancer preceding chemotherapy. Cancer Nursing: 2020, 43(2), p. E71-E78

19. Butow P, Devine R, Boyer M, Pendlebury S, Jackson M, Tattersall MH. Cancer consultation preparation package: changing patients but not physicians is not enough. $\mathrm{J}$ Clin Oncol. 2004;22(21):4401-4409.

20. Albada A, Ausems MG, Otten R, Bensing JM, van Dulmen S. Use and evaluation of an individually tailored website for counselees prior to breast cancer genetic counseling. J Cancer Educ. 2011;26(4):670-681.

21. Brooke J. SUS: a 'quick and dirty' usability scale. In: Jordan P, Thomas B, Weerdmeester B, McClelland I, eds. Usability Evaluation in Industry. London: Taylor \& Francis; 1996:189-194.

22. Bol N, van Weert JC, de Haes HC, et al. Using cognitive and affective illustrations to enhance older adults' website satisfaction and recall of online cancer-related information. Health Commun. 2014;29(7):678-688.

23. Bangor A, Kortum PT, Miller Jt. An empirical evaluation of the system usability scale. Int J Hum Comput Interact. 2008;24(6):574-594.

24. Van Bruinessen IR, van Weel-Baumgarten EM, Gouw H, Zijlstra JM, van Dulmen S. An integrated process and outcome evaluation of a web-based communication tool for patients with malignant lymphoma: randomized controlled trial. J Med Internet Res. 2016;18(7):e206.

25. Brandes K, van der Goot MJ, Smit EG, van Weert JCM, Linn AJ. Understanding the interplay of cancer patients' instrumental concerns and emotions. Patient Educ Couns. 2017;100(5):839-845.

\section{Tables and Figures}

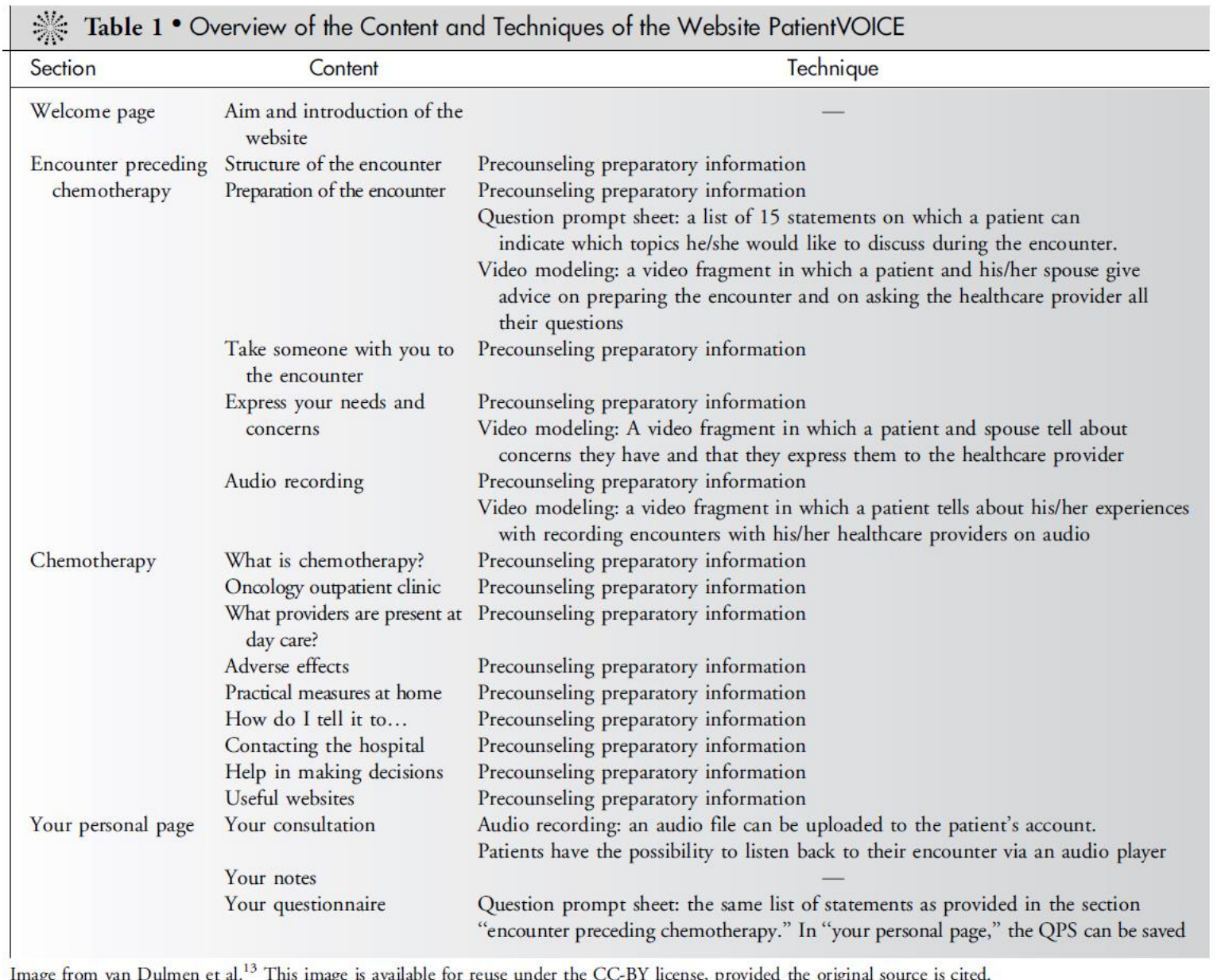


Driesenaar, J.A., Dulmen, S. van, Weert, J.C.M. van, Noordman, J. Patients' evaluation of a preparatory online communication tool for older patients with cancer preceding chemotherapy. Cancer Nursing: 2020, 43(2), p. E71-E78

Figure $\mathrm{n}$ Screenshot of the homepage of the website PatientVOICE. Image from Dulmen van et al.13 This image is available for reuse under the CC-BY license, provided the original source is cited.

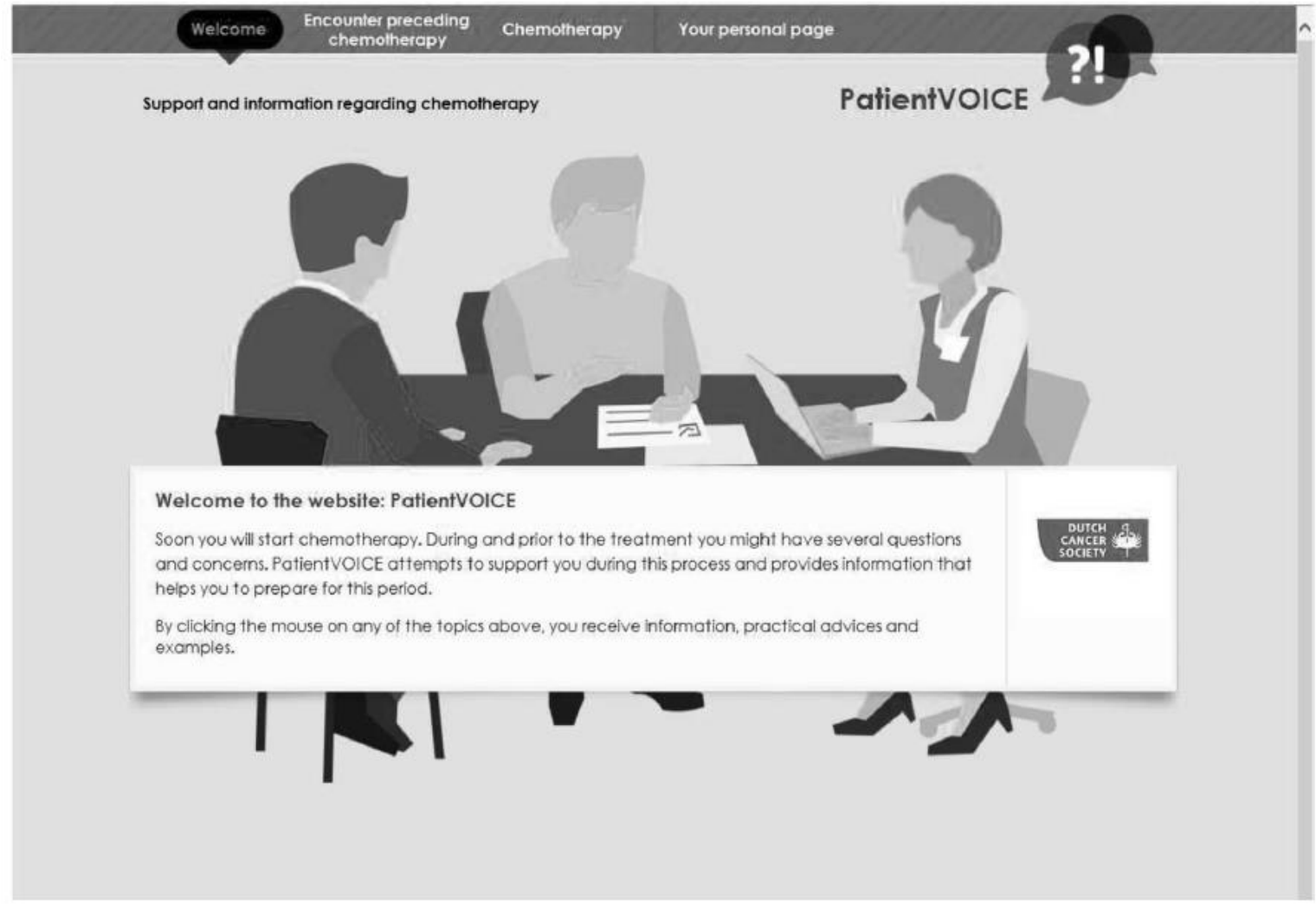


Driesenaar, J.A., Dulmen, S. van, Weert, J.C.M. van, Noordman, J. Patients' evaluation of a preparatory online communication tool for older patients with cancer preceding chemotherapy. Cancer Nursing: 2020, 43(2), p. E71-E78

\begin{tabular}{|c|c|}
\hline Male & $25(56.8 \%)$ \\
\hline Age, mean $\pm S D, y$ & $67.2 \pm 3.04$ \\
\hline \multicolumn{2}{|l|}{ Educational level } \\
\hline Low & $12(27.3 \%)$ \\
\hline Intermediate & $10(22.7 \%)$ \\
\hline High & $22(50,0 \%)$ \\
\hline \multicolumn{2}{|l|}{ Ethnical background ${ }^{\mathrm{a}}$} \\
\hline Dutch & $40(90.9 \%)$ \\
\hline Non-Dutch & $3(6.8 \%)$ \\
\hline \multicolumn{2}{|l|}{ Type of cancer } \\
\hline Breast cancer & $7(15.9 \%)$ \\
\hline Lung cancer & $2(4.5 \%)$ \\
\hline Digestive-gastrointestinal cancer & $18(40.9 \%)$ \\
\hline Gynecological cancer & $3(7.5 \%)$ \\
\hline Hematologic (blood or lymph) cancer & $4(9.1 \%)$ \\
\hline Genitourinary cancer & $9(20.5 \%)$ \\
\hline Skin cancer & $1(2.3 \%)$ \\
\hline Other & $3(7.5 \%)$ \\
\hline \multicolumn{2}{|l|}{ Chemotherapy } \\
\hline Soon first treatment & $0(0.0 \%)$ \\
\hline Currently treated & $12(27.3 \%)$ \\
\hline Treated in the past year & $5(11.4 \%)$ \\
\hline Treated $1-5 y$ ago & $10(22.7 \%)$ \\
\hline Treated $>5 y$ ago & $6(13.6 \%)$ \\
\hline Never been treated & $11(25.0 \%)$ \\
\hline \multicolumn{2}{|l|}{ Computer and tablet use } \\
\hline Daily & $43(97.7 \%)$ \\
\hline Weekly & $1(2.3 \%)$ \\
\hline \multicolumn{2}{|l|}{ Internet use } \\
\hline Daily & $40(90.9 \%)$ \\
\hline Weekly & $4(9.1 \%)$ \\
\hline
\end{tabular}

${ }^{2}$ One missing value. 
Driesenaar, J.A., Dulmen, S. van, Weert, J.C.M. van, Noordman, J. Patients' evaluation of a preparatory online communication tool for older patients with cancer preceding chemotherapy. Cancer Nursing: 2020, 43(2), p. E71-E78

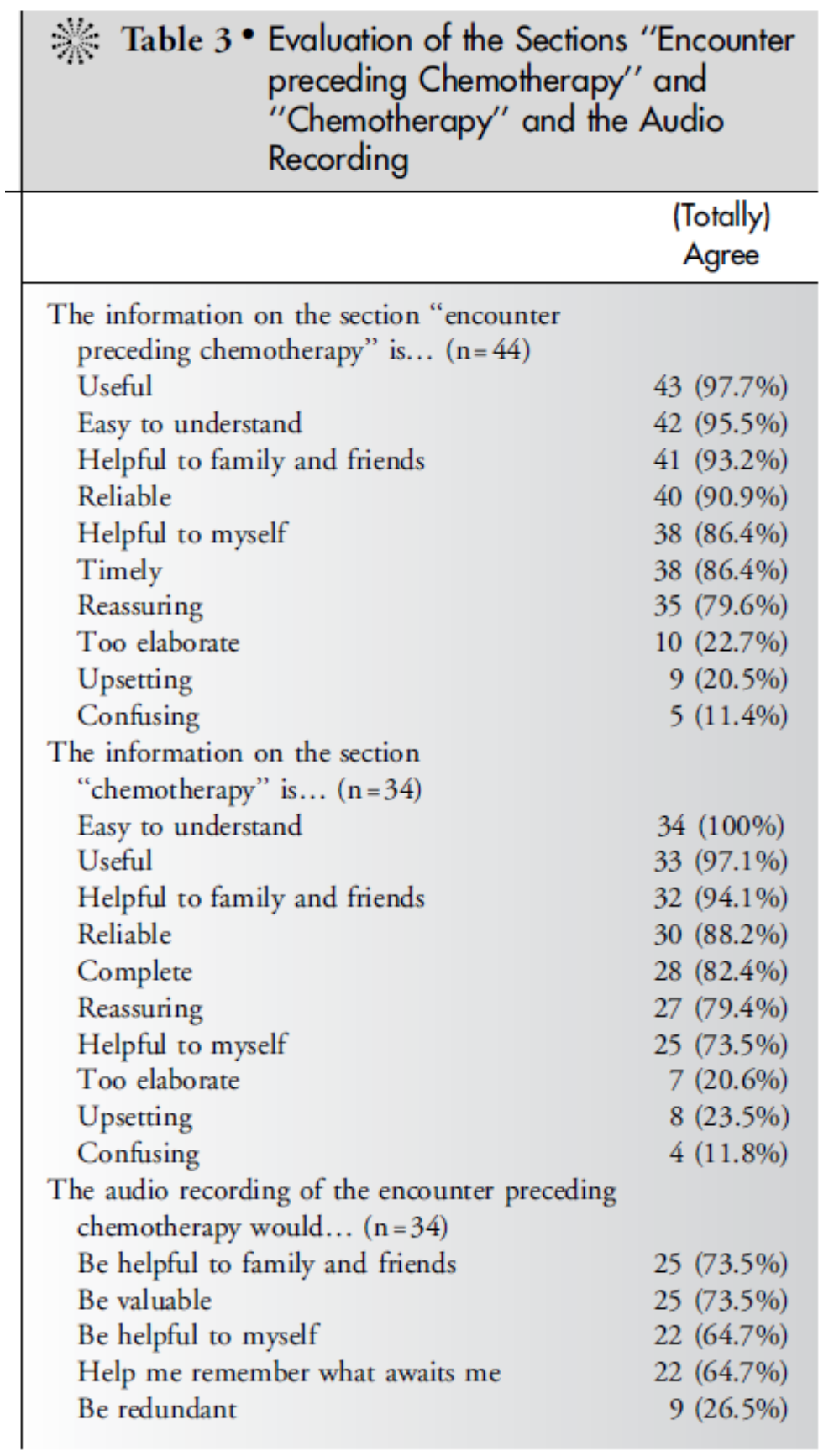


Driesenaar, J.A., Dulmen, S. van, Weert, J.C.M. van, Noordman, J. Patients' evaluation of a preparatory online communication tool for older patients with cancer preceding chemotherapy. Cancer Nursing: 2020, 43(2), p. E71-E78

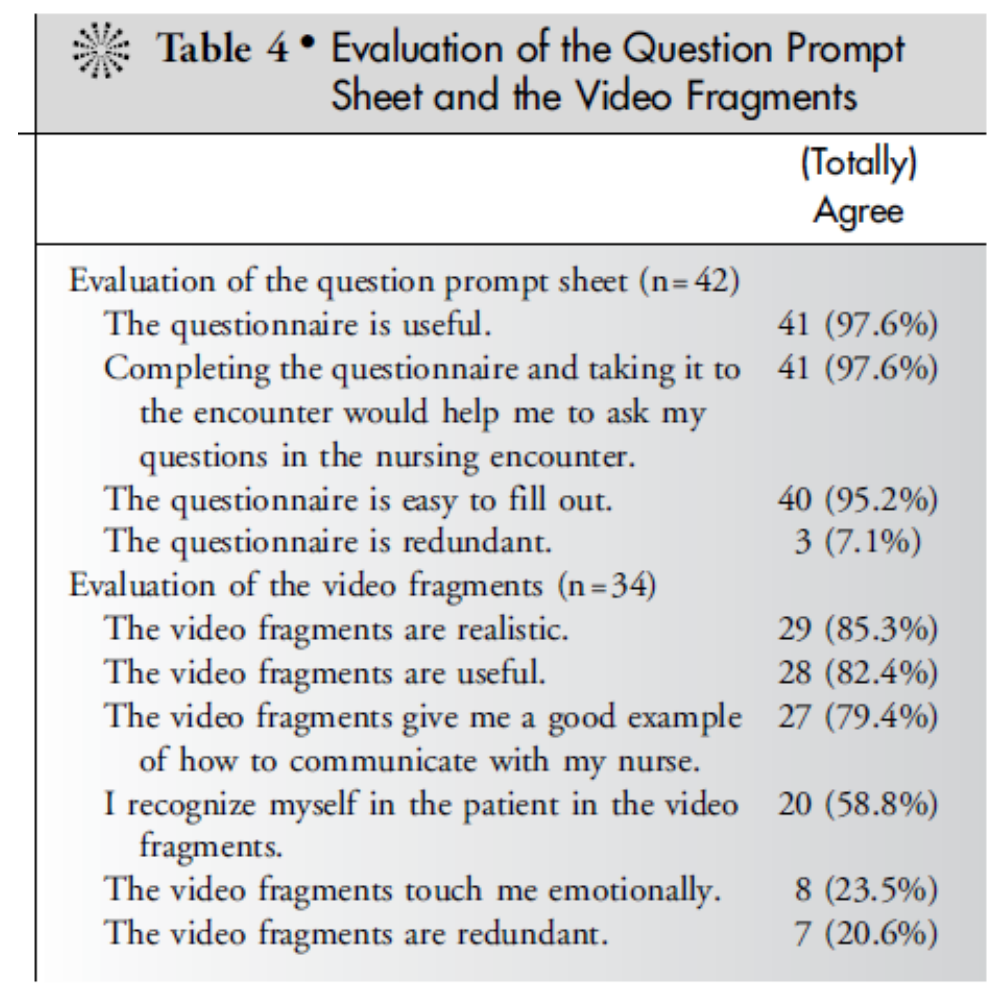

